\title{
Struggling With Recovery: Participant Perspectives on Battling an Eating Disorder
}

\author{
Michelle D'Abundo \\ Pamela Chally
}

Recovery from an eating disorder encompasses a complex matrix of mental, physical, and social factors. Although methodological differences affect rates of reported recovery (from 24 to $76 \%$ ), many women are not experiencing full recoveries from eating disorders. This trend is apparent in persons who discontinue severely destructive behaviors but continue to exhibit psychiatric problems and impairment in social and occupational roles. The purpose of this grounded theory study was to provide an explanatory schema about recovery. Data collection included 17 interviews, a focus group, and participant observation. Findings included repeating wavelike patterns of disease and recovery that seldom returned to a state of normal eating but, instead, remained at a level of atypical eating.

Keywords: eating disorders; eating disorder curve; grounded theory; recovery

$\mathbf{R}$ ecovery from an eating disorder encompasses a complex matrix of mental, physical, and social factors. With intentions of improving treatment and outcomes, professionals from numerous disciplines, including medicine, nursing, nutrition, psychology, sociology, and other allied health professions, have examined the process of recovery in individuals who have eating disorders.

Unpredictable disease course and treatment outcomes are of most significance to health professionals attempting to treat those who already have an eating disorder. Unfortunately, past research has been inconclusive and, therefore, has not identified effective strategies for treatment.

According to Fisher et al. (1995), eating disorders have become the third leading chronic illness among female adolescents. Research on eating disorders indicates that rates of recovery range from 24 to $76 \%$ (Eckert, Halmi, Marchi, Grove, \& Crosby, 1995; Field et al., 1997; Katzman, Golden, Neumark-Sztainer, Yager, \& Strober, 2000; Keel \& Mitchell, 1997; Rollins \& Piazza, 1981; Strober, Freeman, \& Morrell, 1997; Zipfil, Lowe, Reas, Deter, \& Herzog, 2000). Identified differences in rates of recovery are partly due to the definition and criteria for recovery. Most criteria are based on body weight, mortality, symptom severity, standardized assessments, eating attitude, and menstrual cycle (Herzog, Nussbaum, \& Marmor, 1996; Wozniak \& Herzog, 1993). Recovery rates are also influenced by methodological factors, including differences in recovery duration, methods of follow-up, assessment of

AUTHORS' NOTE: This research was funded in part by a university grant on eating disorders.

QUALITATIVE HEALTH RESEARCH, Vol. 14 No. 8, October 2004 1094-1106

DOI: $10.1177 / 1049732304267753$

(C) 2004 Sage Publications 
recovery, differences in sample size, and attrition rates (Herzog, Nussbaum, et al., 1996). Such differences continue to cause disagreement regarding the issue of recovery from eating disorders.

Although methodological differences affect reported rates of recovery, many people are not recovering from eating disorders. This trend is especially apparent in patients who discontinue destructive behaviors but continue to exhibit psychiatric problems and impairments in social and occupational roles (Hoffman \& Halmi, 1993; Steiner \& Lock, 1998). Even those that receive extensive medical monitoring, medications, and treatment are not being cured. Many outcome studies are relatively short-term and suggest promising results. Longitudinal studies, however, are fewer in number and are less positive about complete recovery. Regardless, some patients recover fully, some improve, and others remain affected by the disease.

Methods of treatment for eating disorders vary. After an extensive review of the literature on the epidemiology, diagnosis, and therapy of eating disorders, Walsh, Wheat, and Freund (2000) concluded that the treatment of eating disorders should involve a multidisciplinary approach including primary care providers, nutritionists, and mental health professionals. General immediate treatment for both people with anorexia and those with bulimia should focus on the normalization of eating patterns. In recovery, best results occur with weight restoration and individual and family therapy. Depending on the eating disorder, treatment might include the use of psychotropic medications. In general, the use of medication is more effective in the treatment of bulimia than in the treatment of anorexia.

Although studies have focused on recovery rates and the treatment of eating disorders, few researchers have explored the patient's perspective of recovery. In this research, we will begin to address the gap in the literature by presenting the experiences of women that have struggled to recover from eating disorders.

\section{PURPOSE}

The purpose of this study was to explore the process of recovery in women and girls with eating disorders. The research question was: What are the research participants' perspectives about recovery from eating disorders? This research discloses the participants' perceptions of their illness by providing an explanatory schema about the experience of recovering from an eating disorder.

In this study, we employed grounded theory as the theoretical construct. According to Patton (2002), grounded theory concentrates on producing theory instead of focusing on theoretical content. We collected and analyzed data simultaneously with the intention of constructing a theoretical model. Emerging theoretical concepts were drawn and built on through a constant comparative method (Glaser $\&$ Strauss, 1967). We used grounded theory to identify basic social processes that motivated human behaviors to develop a complex understanding of recovery in women with eating disorders.

\section{RESEARCHERS' PERSPECTIVES}

Eating disorders primarily occur in White middle-to-upper class women and girls, and both researchers were demographically similar to the research population. One 
researcher had a family member who had the disease. The other had struggled with atypical eating as an adolescent. Through literature reviews and previous research experience about eating disorders, we have professional knowledge about recovery from eating disorders in the general population. However, we conducted this project to display the perspectives of the research participants. Therefore, the data collected expanded our preexisting knowledge regarding eating disorders.

\section{METHOD}

\section{Participants}

The participants in this study were women who had recovered from or were in the process of recovering from an eating disorder. Participants ranged in age from 17 to 46 years and included 17 White, 2 Black, and 1 Hispanic woman. Fifteen of the participants were single, 3 were married, and 2 divorced. The educational backgrounds of participants were as follows: One had not finished high school, 1 had a high school diploma, 10 had some college, 5 were college graduates, and 3 held graduate degrees. The participants in this study were in different stages of recovery. Some participants had sought and received professional treatment. However, others had not, because they chose to conceal their eating disorder for personal and professional reasons.

We recruited participants by posting flyers about the study around a midsize Southeastern university campus inviting interested persons to contact us. Women who attended a local health care facility eating disorder support group were also invited to participate. We also used a snowball technique whereby participants assisted in identifying other possible research participants.

With institutional review board approval, we explained the research purpose to participants and obtained consent with the understanding of confidentiality. Because medical records were not obtainable, we relied on the participants' selfreported incidence of an eating disorder. The research was not limited to those who had been clinically diagnosed as having an eating disorder, because many women hide their eating disorder and therefore remain undiagnosed by health professionals. If the participant identified that her quality of life had been significantly affected by problems with eating, she was eligible for participation in the study. The interviewees, focus group members, and support group attendees participating in the study included both women with anorexia and those with bulimia who identified that they had made positive progress in their struggles with their eating disorder. It is important to note that many participants described symptoms of both anorexia and bulimia and thus were not referenced in this analysis as anorexic or bulimic.

\section{Data Collection}

Because of the complex nature of recovery from eating disorders, it was necessary to triangulate methods of data collection. The project included in-depth semistructured interviews, participant observation at a support group for persons with an eating disorder, and a focus group. Seventeen women participated in informal, 
semistructured interviews that were based on an interview guide. Interviews lasting between 1 and 2 hours were audiotaped and later transcribed verbatim.

Participant observation involved attending a weekly support group for 9 months. Notes and memos were written immediately after the meetings. The meetings were held for $1 \frac{1 / 2}{2}$ hours each Monday evening at a local health care facility. Group size ranged from 4 to 10 participants, with an average of 5 to 6 persons each week. The group was a spin-off of support groups sponsored by the National Association of Anorexia Nervosa and Associated Disorders. The general format involved asking participants what they would like to discuss that evening and building discussions based on the desires of the group.

We jointly conducted a focus group, with one of us serving as facilitator and the other as co-facilitator. The facilitator primarily served as the moderator, whereas the co-facilitator took notes and handled equipment. Five women, 2 of whom were interviewed previously, were recruited by posting flyers on campus. The focus group, which was audiotaped and transcribed verbatim, focused on the participants' experiences with recovery. Immediately after the focus group, we held a debriefing session to compare impressions and notes, and to record important trends in the discussion. We held the focus group after the interviews were conducted, and it was useful in the clarification of data categories and concepts.

This research indicated that the experience of an eating disorder was different for each individual. Depending on the etiology, process, and outcome of the disorder, the participants struggled with different issues. Therefore, saturation while investing the topic of eating disorders might never occur. However, in this study, in which we focused primarily on the process and experience of recovery, there were similarities in the experiences of participants. As the research progressed, there was some redundancy. Thus, the sample seemed adequate to address the purpose of this research, which was to explore the participants' perspectives of recovery from an eating disorder.

\section{Data Analysis}

Data analysis and collection regarding recovery occurred simultaneously based on the constant comparative method (Glaser \& Strauss, 1967; Hutchinson, 1993). We coded data line by line and then assigned them to categories. The categories were revised as more information was collected and emerging concepts were sharpened. Specifically, we looked for the social processes of participants relating to recovery from their eating disorder. Emerging concepts suggested the need for information to be sought in subsequent interviews, and we modified interview questions as the focus of the analysis sharpened.

Emerging concepts led to the development of a model of behavior displaying the relationships of categories. We also explored contradictory cases and adjusted the model to represent all of the interview participants' experiences accurately. The developing theory encompassed significant behavioral variation identified in the participants interviewed. Further coding and questioning of the data, along with additional interviews, facilitated the generation of the processes described in the findings.

The validity of this study was strengthened by the aforementioned data analysis techniques and triangulation of methods. We used peer examination, whereby 
both researchers and a graduate assistant coded data separately and then compared results. We applied the constant comparative method through hypothesis testing, revision of conceptual categories, and the inclusion of deviant cases.

\section{FINDINGS}

The data generated a cyclical model of an eating disorder referred to as the eating disorder curve. The eating disorder curve illustrates how eating disorders developed and became more severe, and how the process of recovery began and continued in the research participants in this study. Imagine eating prior to the onset of an eating disorder as a flexible line. As the eating disorder developed, the line swelled and created an elevated, wavelike formation. When the eating disorder subsided, it returned to a flexible line. Relapses in recovery occurred and were illustrated in this model by elevated waves. The components of the eating disorder curve included increasing severity, the circle of acceptance, and decreasing severity, and will be described in further detail in the following section.

\section{Increasing Severity of Eating Disorder}

In this study, the development of the disorder usually began with unhealthy attitudes favoring thinness, which sparked dieting, bingeing, or purging. The development of unhealthy attitudes and behaviors started the incline on the slope to increasing severity of the eating disorder. One participant summarized society's attitude regarding weight and appearance by saying, "You always think, well if I'm skinny, I'm happy. If I'm thinner, I'm happy. Well, I'm not happy ... I've always wanted to be thinner." As the eating disorder developed, issues related to eating increasingly consumed the participants' thoughts, and the severity of the disorder continued to escalate.

The goal of extreme thinness, combined with a quest for perfectionism, resulted in behaviors associated with eating disorders. The participants became obsessed with weight loss, and as a result, the severity of the eating disorder increased and the slope climbed and became steeper. Analysis of the data led to the identification of three processes: thinking irrationally, struggling for control, and withdrawing from society.

The presence of irrational thinking was identified in the data. Research participants used terminology such as "irrational," "negative mind," or "crazy thinking" to describe their thinking while battling the disorder. Negative thinking was displayed in the participant quotes such as, "I thought in my head that everyone else was against me. They were telling me to gain weight ... because they were against me," and "It was like voices, but it is not voices. It is myself, my two selves. One has no self-esteem and the other is secure."

Struggling for control accompanied irrational thinking, as participants described in detail that control was significant in the etiology of their eating disorder. For example, women interviewed expressed feeling out of control in all areas of their lives. The most immediate and convenient coping mechanism was the control involved in their relationship to eating. A participant spoke of her experience, "I had power over nothing in my life, but I could control that number [my weight]. 
That's one thing I truly had power over." Also describing control another participant stated, "It was a control thing ... I wasn't very pretty. I wasn't very popular. This was one thing I could control. I could make myself thinner."

Participants exhibited a tendency to withdraw from society. Preoccupation with food and weight created a barrier between participants, family, and others. The disorder limited their ability to concentrate on other important issues, such as school or work, and led to social isolation in attempts to hide their eating behaviors. A focus group participant said, "My boyfriend of three years doesn't know. This is the person I'm going to marry." Other participants indicated that their preoccupation with food and other obsessions related to their disease did not leave them time for anything else and contributed to increased periods of isolation. Other quotes displaying the tendency toward isolation include, "I didn't want to leave my house. I didn't want to bother with people," and "I put up the attitude like I didn't want to talk to you, just go away." Social withdrawal, along with irrational thinking and issues of control, collectively contributed to the increasing severity or incline slope of the eating disorder curve.

With the continuation of the eating disorder, data showed that the problem escalated until it reached a pinnacle, often followed by a turning point. The pinnacle of the disease was defined as the most severe point of the disease, wherein both mental and physical symptoms threatened the health of the participant. The pinnacle of the disease was represented by the highest point of the wave on the eating disorder model. This point was identified by an extreme imbalance, with the disorder having control over most aspects of the participants' lives. In cases with positive outcomes, the turning point resulted in improvement in symptoms associated with the disorder, represented by the declining slope of the eating disorder cycle. The quality of the participants' health increased as the disease, involvement of others, and spirituality were accepted. The turning points shared by participants were events or people that acted as catalysts to recovery.

\section{Circle of Acceptance}

We have used the circle of acceptance to display the point following increasing severity of the disease wherein social, mental, and medical implications of the disease interfaced. This point described the pinnacle of the disease and the highest point on the eating disorder curve, at which mental and physical symptoms were most serious. The pinnacles of research participants were similar and extremely important to recovery, as this was the point at which the disease either improved or remained at the highest level of severity. Those suffering from chronic clinically defined eating disorders stay at their pinnacle for extended periods of time. Fortunately, the research participants interviewed in this study did not stay at the pinnacle of their disease. Rather, participants' conditions gradually improved, as displayed in the circle of acceptance.

The circle of acceptance model shows how elements of recovery worked together to initiate steps toward wellness for participants in this study. The three areas of the circle of acceptance included acceptance of the disease, spirituality, and relationships. The interaction of the three areas of the circle of acceptance seemed to contribute to the individuals' feelings of self-worth. We drew the areas of acceptance on a circle to display their continuous and circular nature (Figure 1). Although 


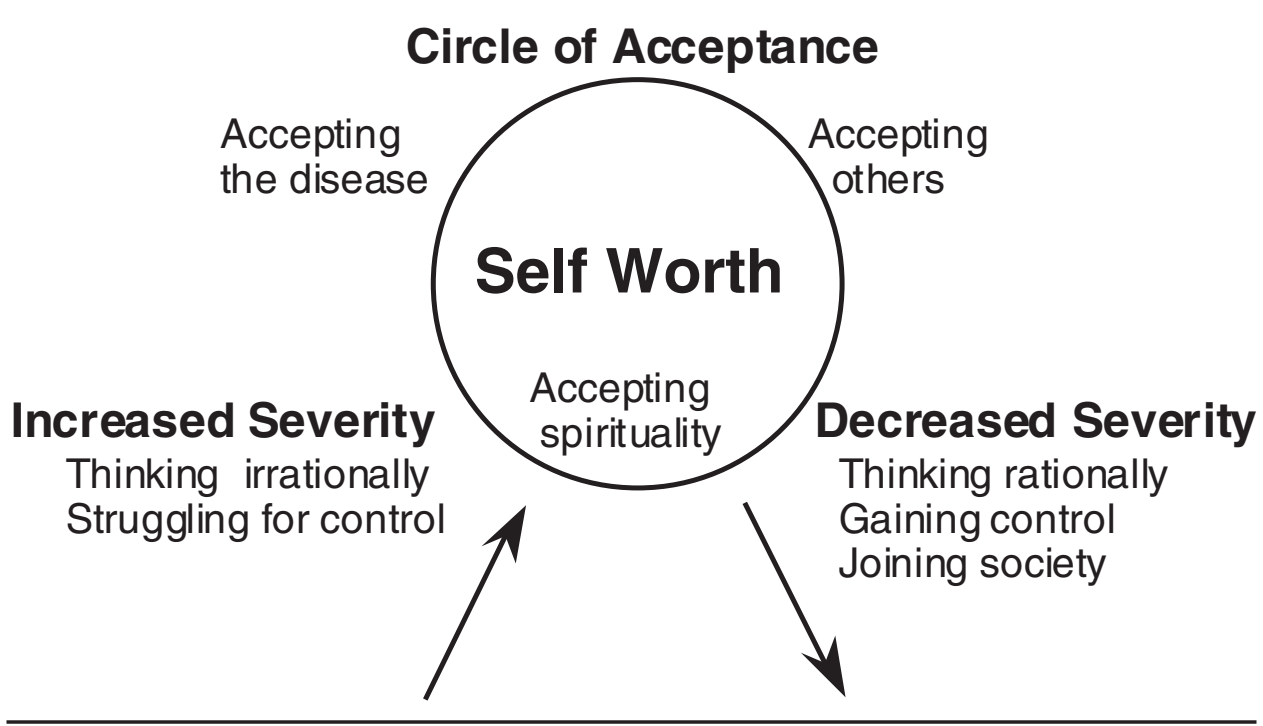

FIGURE 1: $\quad$ Eating Disorder Curve

all of the participants' recoveries involved the entire circle of acceptance, one area of the circle might have been more significant to the recovery of a particular participant.

For many, acceptance of the disease was triggered by a turning point or critical event that resulted in the recognition of the severity of their eating disorder. When faced with hospitalization, a focus group member said, "I work in a hospital. I was not about to be admitted to one." She felt she would rather change her behaviors than have her colleagues know that she was suffering from an eating disorder. For others, the turning point of their disease was unrelated to the eating disorder. For example, one participant was in a car accident and realized the value of life after being faced with death.

Acceptance of the disease also involved recognizing negative consequences of the eating disorder. Participants realized effects of the eating disorder included looking bad, being cold, being embarrassed about eating behaviors, bingeing, purging, fertility problems, not thinking clearly, feeling tired all the time, and having no social life. Participants similarly described "getting fed up" with the consequences of the eating disorder.

The most influential factors leading to the acceptance of the disease were medical conditions related to the eating disorder. Most participants disclosed that the disorder had affected their health negatively. Some interviewees talked about a situation that really scared them, such as passing out, ulcers, cardiac arrest, or severe esophageal bleeding. For example, a participant said, "I collapsed on the beach ... and I basically accepted that this is not healthy." A research participant with bulimic tendencies recalled her heart beating irregularly and extremely quickly when she purged. Other participants experienced severe stomach cramps and spontaneous vomiting. Medical problems were often involved in the acceptance of the disease through recognition that they were affecting current and future health outcomes. 
Acceptance of spirituality was the second area of the circle. Components of spiritual health include having purpose and higher meaning in life (Hawks, 2004). Within the circle of acceptance, spirituality consisted of having hope, valuing life, and trusting in God.

Recovery in research participants was enhanced through hope for the future and value of life. Those interviewed began to want a life beyond what they were experiencing with the eating disorder and, in the process, started to look forward to college, marriage, having children, and spending time with family. Two participants expressed their desire to be role models for young women. For example, "I was working with young girls ... and didn't want them to feel the way that I did about my size." Another participant taught gymnastics and educated her students' parents about the dangers of emphasizing thinness. Some participants talked about the value of life and wanted to prevent others from the devastating effects they had experienced as a result of their own eating disorders.

Acceptance of spirituality also involved trusting in a higher power. Many participants believed their faith essential to the process of recovery. One young woman talked about giving up bulimia for Lent. "As strange as that may sound, I just did it for God during Lent." Others felt establishing a relationship with a higher being allowed them to recognize that their behaviors were self-destructive and detrimental to their health. A woman interviewed asked her God for the faith to take away the eating disorder. The following quote is a discussion with her mother:

I said "I've got this eating disorder and I don't know what to do with it. I can't make
it stop." I said, "Mom, I don't even have the faith to ask God to take it." And she said
"Ask God for the faith to ask Him to take it." I did and I haven't purged since then.
It's like a miracle and how do you give that to anybody on the street that has bulimia
that wants to get rid of it? It's like, give it to God. I mean, it sounds so insane and irra-
tional but what He did is he gave me the faith to suggest that I didn't have to have
it... He would take it just like He would take it if I had cancer... So that is how I got
better.

Acceptance of spirituality enabled participants to accept themselves and their bodies and begin to establish self-worth.

The circle also included acceptance of a relationship with another person. Some participants talked about the significance of their relationship with their counselor. However, most participants in this study relied on more long-term, in-depth relationships. Acceptance of a relationship with a significant other or family member was found to be very important in the participants' recoveries. A relationship with a special individual was more common in the process of recovery from an eating disorder than working with a professional counselor. These relationships were essential to recovery, because they provided unconditional love, support, trust, inspiration, and hope. Although counseling was helpful to some, affirmation of self by another individual important to the person with an eating disorder was found more essential to recovery. The person identified was most frequently a mother, significant other, or father. This is an interesting finding, because often parents or significant others are identified as contributing to the existence of the eating disorder.

All of the factors involved in the three areas of acceptance seemed to occur at similar times in the recovery period. The areas appeared to work together to build participants' self-worth. As the self-worth was enhanced, the severity of the disor- 
der decreased. Thus, the circle of acceptance appeared to motivate the initiation of the process of recovery.

Completion of the circle of acceptance was especially apparent in participants who have made the most progress in their struggle with recovery. Participants who had completely ceased negative behaviors associated with the disorders had the most complete circles. For example, a focus group member said she was no longer bingeing and purging and was somewhat comfortable with her body. She exhibited a complete circle of acceptance that included establishing a relationship with her mother, trusting in God, and accepting that her eating disorder behaviors needed to be treated. Completing the circle of acceptance enabled the participant to recognize her own unique value and therefore promoted the initiation of self-worth. As her self-worth increased, the participant was able to continue a successful process of recovery.

Some participants did not exhibit a full circle of acceptance. Those research participants that admitted to occasionally practicing behaviors associated with the disorder were often missing one area in the circle of acceptance. For example, one participant began to accept spirituality in the form of establishing a relationship with God and accepted that she suffered from an eating disorder. However, she did not feel that she had support from others. The incomplete circle prohibited her from ceasing to purge once or twice a month.

\section{Decreasing Severity of Eating Disorder}

The circle of acceptance was followed by a decline in the severity of the disease, which we have represented by a descending slope on the eating disorder curve. This decline represented movement toward healthy behaviors. Participants started to think rationally, gain control, and rejoin society. Once the circle of acceptance began, the eating disorder took on less significance. When rational thinking was initiated, research participants realized that eating was necessary for survival. An interviewee expressed, "Thinking that you could keep one thing down at dinner and live is very irrational. I know now that you cannot deal with problems that way." The participants began to see their bodies realistically and were able to identify extreme thinness and unhealthy behaviors as the cause of the negative mental, physical, and social effects that they had experienced. In addition, participants moved beyond extreme emphasis on outward appearances and recognized that self-worth could be gained through other aspects of their being.

Gaining control was necessary in the process of recovery from an eating disorder. The women in this study had a false sense of control, as described by a participant who said, "I thought by not eating I was in control. I suddenly realized that when I eat, I gain control." Physical, mental, and social issues that are important to most healthy people gained relevance in their lives. A participant said, "When you quit eating, you are really out of control. Now I eat healthy and am in control of my life!"

Negotiating conditions of weight gain, for example, how quickly one will gain weight, how one will give up obsessions with food, or how one will substitute exercise for bingeing and purging, were examples of control playing a significant role in the recovery process. The need to be involved in decisions regarding recovery was apparent in participants. One interviewee described how work with her therapist 
was "making an agreement ... I got to have a little bit of control and there was compromise." She continued by telling the researcher how her meal plan was negotiated. The therapist did not ignore the fact that she did not live up to the food plan but worked with her to gradually increase her fat and calorie intake. A similar approach was used in regard to exercise, as displayed in the statement "She wanted to exercise everyday for an hour. He [the therapist] compromised to three times a week."

As the disease decreased in severity, the women interviewed described how they rejoined society. They began to build relationships with a significant other and/or friends and family members. Socializing became something they looked forward to as they felt comfortable participating in activities. Even though it was challenging to go out to restaurants and eat with others, they began to do so. A participant related,

Now I'm finding other things to find joy in. It may be watching a movie with a friend or spending time with my family. I realize how much fun I have with my friends and how much I like to be alive.

Joining society, thinking rationally, and gaining control enabled participants to overcome the debilitating effects of their eating disorder and move toward a healthier lifestyle.

Many of the participants' behaviors associated with the disorder ceased as they proceeded along the downward slope of the cycle. However, participants still experienced residual effects, such as unhealthy attitudes, restricted eating, and obsession with weight and appearance. The continued existence of factors associated with the eating disorder increased the chance that eating disorder would resurface. Unfortunately, a number of participants did experience relapses and went through the cycle more than once in their lives.

\section{DISCUSSION}

Data indicated that the cyclical process of an eating disorder included increasing severity of the disease as evidenced by thinking irrationally, struggling for control, and withdrawing from society. The research concept, termed the circle of acceptance, described the process of gaining self-worth. This process occurred through acceptance of the disease, acceptance of spirituality, and acceptance of others. After the circle of acceptance began, participants demonstrated decreasing severity of the disease by thinking rationally, gaining control, and joining society.

The circle of acceptance model proposed in this study involves issues of selfworth, including acceptance of the eating disorder, others, and spirituality. The concept of accepting the disease has been minimally explored in research and literature on eating disorders. In this study, others refers to family and friends who were involved in the recovery of research participants. Research exploring social relations and recovery from bulimia by Rorty, Yager, Buckwalter, and Rossotto (1999) indicated that relationships with relatives were difficult for participants. However, regarding the role of others in the recovery process, they explained, "That relationships with friends were less problematic for remitted than actively bulimic subjects hints that building solid relationships with friends may be critical to healing" 
(p. 10). Issues of personality and affective disorders like control and irrational thinking evident in this study might lead to social isolation. Therefore, it seems that attention to development and maintenance of relationships with others is an important aspect of recovery for people with eating disorders.

In Garrett's (1996) study, similar factors to the circle of acceptance are discussed in the recovery from anorexia. Garrett described the participants' recovery as a spiritual quest involving connections between inner self, others, and nature. Garrett emphasized the need to recognize spirituality of recovering clients and to encourage connections between mind and body. In a review of the literature on spirituality and physical health, Mueller, Plevak, and Rummans (2001) found, "Most studies have shown that religious involvement and spirituality are associated with better health outcomes, including greater longevity, coping skills, and health-related quality of life (even during terminal illness) and less anxiety, depression, and sui-

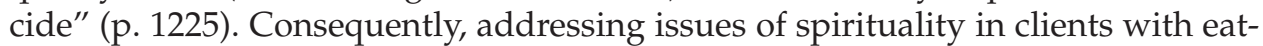
ing disorders seems to be an inexpensive approach to a very complex, costly, and difficult-to-treat illness.

Participants indicated recovery from an eating disorder was a continuous struggle. In Garrett (1997), research participants defined recovery not as a final point but always as an ongoing process. Although some participants discontinued destructive behaviors, many displayed a continued preoccupation with physical appearance, eating, and food. Statements such as "I'm not over it," "I'm not completely better," and "It's always in the back of my mind" were expressed by participants. The long-term effects and continuous nature of eating disorders has been identified by long-term follow up studies by Eckert et al. (1995); Herzog, Dorer, et al. (1999); Keel, Mitchell, Miller, Davis, \& Crow (1999); and Strober et al. (1997). Therefore, research indicates that both anorexia and bulimia nervosa are characterized by partial recovery with some relapse.

Although some participants met the criteria for full recovery, this research indicated that the participants' quality of life continued to be affected by a less severe form of the eating disorder. Many participants made significant gains in their battles against their eating disorders, but few of the participants in this study had "fully recovered," as they voiced that the disorder still affected their lives. Participants in this study described restricting and/or bingeing behaviors, as well as other behaviors indicating residual effects of the eating disorders.

This study has important practical applications. First, the importance of listening and recognizing the experiences of people who have recovered from eating disorders is displayed in this research. Based on these findings, health professionals must recognize that meeting the diagnostic criteria for recovery does not mean that the patient is cured. From this research, it seems eating disorders are chronic conditions that will affect people with the disease throughout their lives. Ideally, efforts should focus on preventing eating disorders before they begin. However, research remains inconclusive regarding the effectiveness of prevention programs. Therefore, the second best plan of action when dealing with eating disorders is early detection. Thus, it is of primary importance that health and medical professionals be aware of the symptoms of eating disorders and skilled in methods of intervention. In the meantime, researchers need to focus on developing effective prevention programs to avoid the onset of the eating disorder.

The proposed concept of the circle of acceptance describes the experience of the participants in this study. Future research is needed to examine how the circle of 
acceptance model, including acceptance of the disease, others, and spirituality, can be used to improve recovery rates and the effectiveness of treatment programs. The factors identified might also play a significant role in the initiation and course of eating disorders. Therefore, research should be long-term with periodic follow-ups to understand the entire process of an eating disorder from onset to conclusion.

In conclusion, the chronic nature of eating disorders requires treatment programs and health professionals to focus on helping patients to identify that they are suffering from a disease. Patients need to understand that their eating disorder is a combination of negative health behaviors but does not define their self-worth. Anorexic or bulimic individuals must determine their own self-concept independent of their eating disorder. Through continued exploration of issues discussed in this research, treatment programs and health professionals might improve recovery rates and the quality of life for people battling eating disorders.

\section{REFERENCES}

Eckert, E. D., Halmi, K. A., Marchi, P., Grove, W., \& Crosby, R. (1995). Ten-year follow-up of anorexia nervosa: Clinical course and outcome. Psychological Medicine, 25, 143-156.

Field, A. E., Herzog, D. B., Keller, M. B., West, J., Nussbaum, K., \& Colditz, G. A. (1997). Distinguishing recovery from remission in a cohort of bulimic women: How should asymptomatic periods be described? Journal of Clinical Epidemiology, 50(12), 1339-1345.

Fisher, M., Golden, N. H., Katzman, D. K., Kreipe, R. E., Rees, J., Schebendach, J., et al. (1995). Eating disorders in adolescents: A background paper. Journal of Adolescent Health, 16, 420-437.

Garrett, C. J. (1996). Recovery from anorexia nervosa: A Durkheimian interpretation. Social Science $\mathcal{E}$ Medicine, 43(10), 1489-1506.

Garrett, C. J. (1997). Recovery from anorexia nervosa: A sociological perspective. International Journal of Eating Disorders, 21, 261-272.

Glaser, B. G., \& Strauss, A. I. (1967). The constant comparative method of qualitative analysis. Hawthorne, NY: Aldine.

Hawks, S. (2004). Spiritual wellness, holistic health, and the practice of health education. American Journal of Health Education, 35(1), 11-16.

Herzog, D. B., Dorer, D. J., Keel, P. K., Slewyn, S. E., Ekeblad, E. R., Flores, A.T., et al. (1999). Recovery and relapse in anorexia and bulimia nervosa: A 7.5-year follow-up study. Journal of American Academy of Child and Adolescent Psychiatry, 3(7), 829-837.

Herzog, D. B., Nussbaum, K. M., \& Marmor, A. K. (1996). Comorbidity and outcome in eating disorders. Psychiatric Clinics of North America, 19(4), 843.

Hoffman, L., \& Halmi, K. (1993). Psychopharmacology in the treatment of anorexia nervosa and bulimia nervosa. Psychiatric Clinics of North America, 16(4), 767-778.

Hutchinson, S. (1993). Grounded theory: The method. In P. L. Munhall \& C. O. Boyd (Eds.), Nursing research: A qualitative perspective (pp. 180-212). New York: National League for Nursing.

Katzman, D. K., Golden, N. H., Neumark-Sztainer, D., Yager, J., \& Strober, M. (2000). From prevention to prognosis: Clinical research update on adolescent eating disorders. Pediatric Research, 47(6), 709712.

Keel, P. K., \& Mitchell, J. E. (1997). Outcome in bulimia nervosa. American Journal of Psychiatry, 154(3), 313321.

Keel, P. K., Mitchell, J. E., Miller, K. B., Davis, T. L., \& Crow, S. J. (1999). Long-term outcome of bulimia nervosa. Archives of General Psychiatry, 56, 63-69.

Mueller, P. S., Plevak, D. J., \& Rummans, T. A. (2001). Religious involvement, spirituality and medicine: Implications for clinical practice. Mayo Clinic Proceedings, 7(12), 1225-1235.

Patton, M. Q. (2002). Qualitative research and evaluation methods (3rd ed.). Thousand Oaks, CA: Sage.

Rollins, N., \& Piazza, E. (1981). Anorexia nervosa: A quantitative approach to follow-up. Journal of the American Academy of Child Psychiatry, 20, 167-183. 
Rorty, M., Yager, J., Buckwalter, J. G., \& Rossotto, E. (1999). Social support, social adjustment, and recovery status in bulimia nervosa. International Journal of Eating Disorders, 26, 1-12.

Steiner, H., \& Lock, J. (1998). Anorexia nervosa and bulimia nervosa in children and adolescents: A review of the past 10 years. Journal of American Academy of Child and Adolescent Psychiatry, 37(4), 352359.

Strober, M., Freeman, R., \& Morrell, W. (1997). The long-term course of severe anorexia nervosa in adolescents: Survival analysis of recovery, relapse, and outcome predictors over 10-15 years in a prospective study. International Journal of Eating Disorders, 22, 339-360.

Walsh, J. M., Wheat, M. E., \& Freund, K. (2000). Detection, evaluation, and treatment of eating disorders: The role of the primary care physician. Journal of General Internal Medicine, 15(8), 577-590.

Wozniak, J., \& Herzog, D. B. (1993). The course and outcome of bulimia nervosa. Child and Adolescent Psychiatric Clinics of North America, 2(1), 109-127.

Zipfil, S., Lowe, B., Reas, D. L., Deter, H., \& Herzog, W. (2000). Long-term prognosis in anorexia nervosa: Lessons from a 21-year follow-up study. The Lancet, 355, 721-722.

Michelle D'Abundo, Ph.D., M.S.H, C.H.E.S, is an assistant professor in the Department of Health, Physical Education and Human Performance, Salisbury University, Maryland.

Pamela Chally, Ph.D., R.N., is a professor and dean of the College of Health, University of North Florida, Jacksonville. 